\title{
Onychomatricoma of the Nail Bed
}

\author{
Cristina Diniz Borges Figueira de Mello ${ }^{a}$ Leandro Fonseca Noriegab \\ Nilton Gioia Di Chiacchio ${ }^{\text {b, c }}$ Jorge Ocampo-Garzac, d Nilton Di Chiacchiob \\ ${ }^{a}$ Dermatology Department, Universidade Estadual de Campinas, Campinas, Brazil; ${ }^{b}$ Dermatology Clinic, Hospital do \\ Servidor Público Municipal de São Paulo, São Paulo, Brazil; ' Dermatology Clinic, Hospital da Faculdade de Medicina \\ do ABC, São Paulo, Brazil; d Dermatology Department, University Hospital Dr. José Eleuterio González, Universidad \\ Autónoma de Nuevo León, Monterrey, Mexico
}

\section{Established Facts}

- Onychomatricoma is a benign tumor of the nail complex.

\section{Novel Insights}

- We describe a case of onychomatricoma with important involvement of the nail bed.

\section{Keywords}

Nail bed $\cdot$ Nail tumor $\cdot$ Onychomatricoma

\section{Abstract}

Onychomatricoma is a rare and specific benign tumor of the nail complex, with uncertain etiology. The avulsion of the nail plate reveals cavitations and orifices in its proximal extremity. These are associated with villous tumor formations generating digitiform projections at the nail matrix - typical intraoperative findings. We report a rare case of onychomatricoma in which the nail bed was predominantly affected and show evidence of its clinical, intraoperative, and histopathological presentation. The various descriptions of the disease report that its origin is restricted to the nail matrix, leading to secondary changes in the nail plate.

(c) 2018 S. Karger AG, Basel

\section{KARGER}

(C) 2018 S. Karger AG, Basel

E-Mail karger@karger.com

www.karger.com/sad

\section{Introduction}

Onychomatricoma is a rare, specific benign tumor of the nail complex. Since its first description in 1992, some cases have been reported in the literature, describing its clinical, intraoperative, and histopathological characteristics in the nail matrix and plate $[1,2]$. Recent data consider it the result of a cell differentiation disorder at the nail matrix, but with uncertain etiology $[3,4]$.

The incidence of onychomatricoma is higher in Caucasian women at their 5 th decade of life. Fingers are more commonly affected than toes, with prevalence of 63 and $36 \%$, respectively [5]. Although showing characteristic clinical and intraoperative signs, the lack of knowledge about this disease and its tendency to be asymptomatic contribute to a mistaken and/or late diagnosis $[2,5]$.

The combination of thickening, splinter hemorrhages, and xanthonychia of the nail plate represents the 


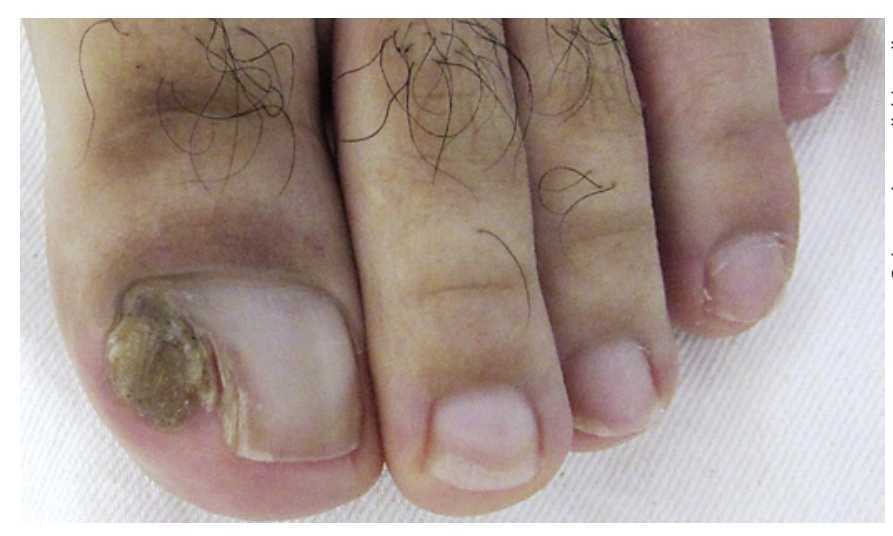

Fig. 1. Dystrophy, hyperkeratosis, longitudinal ridges, yellowish band, and thickened nail plate in the lateromedial region of the left hallux.

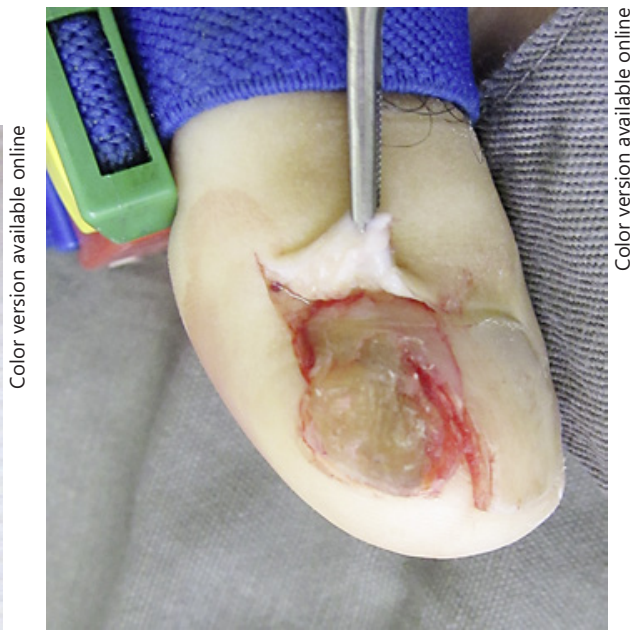

Fig. 2. Intraoperative view after dissection of the proximal nail fold.
Fig. 3. a Ventral face of the nail plate: parallel cavitations and orifices, a pathognomonic sign of onychomatricoma. b Intraoperative view after excision of the nail plate, showing digitiform projections of the matrix as well as the proximal and medium nail bed.
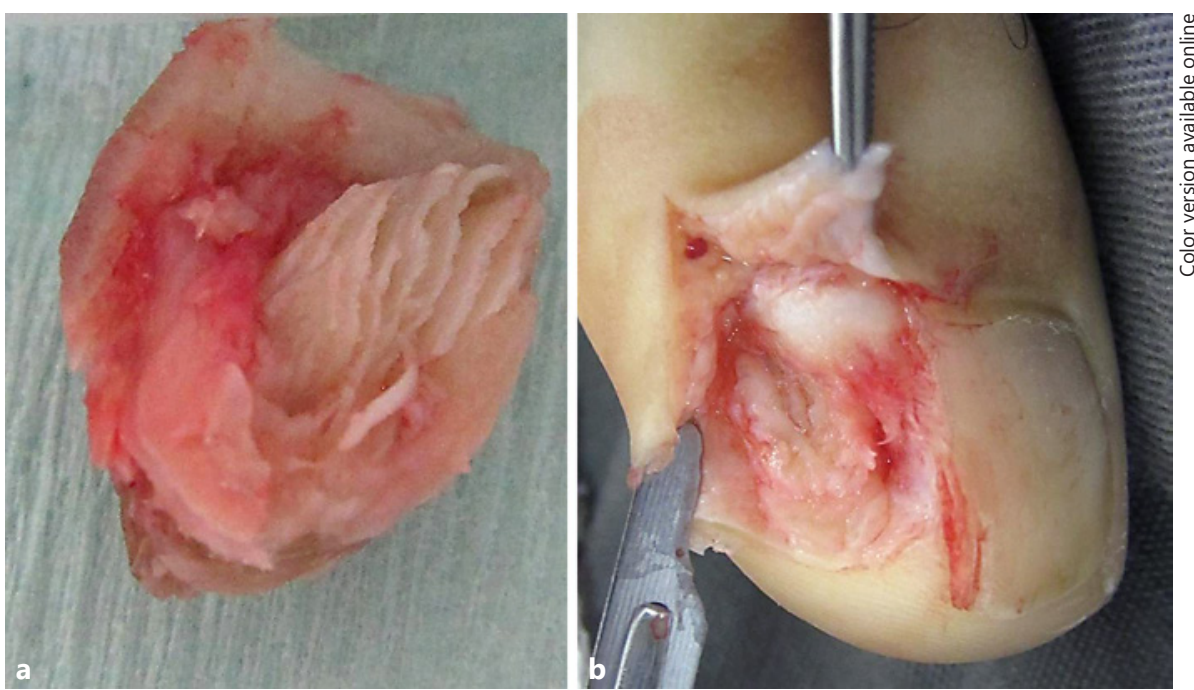

most common clinical presentation, observed in a little more than $50 \%$ of cases. Other findings that may be present include transverse hypercurvature, longitudinal hypercurvature, erythema and edema of the proximal nail fold, prominent longitudinal groove, longitudinal melanonychia, onychodystrophy, and dorsal pterygium $[2,5,6]$.

The ventral view of the nail plate, following its avulsion, reveals cavitations and orifices in its proximal extremity. At the nail matrix, villous tumor formations generating digitiform projections are observed, considered classical of onychomatricoma $[5,6]$.
Dermoscopy, nail clipping, and imaging may help with the diagnosis. However, the gold standard diagnostic method is histopathological examination $[2,5]$. The latter reveals a fibroepithelial tumor with biphasic growth that shows multiple digitiform projections coated by matrix papillomatous epithelium, which runs towards the nail plate and generates perforations $[2,5,7]$.

We report a rare case of onychomatricoma in which the nail bed was predominantly affected and show evidence of its clinical, intraoperative, and histopathological presentation. 


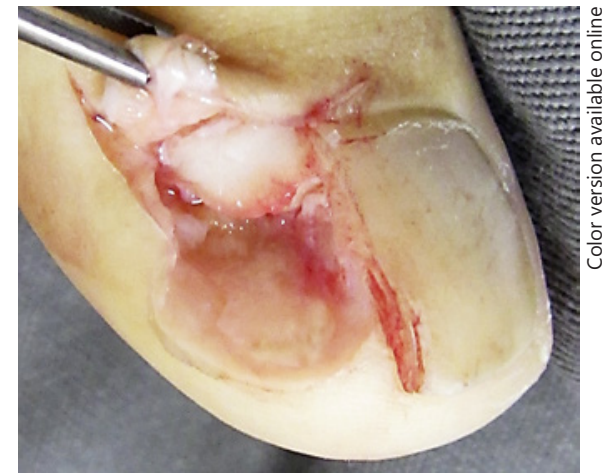

Fig. 4. Intraoperative view after resection of the tumor.

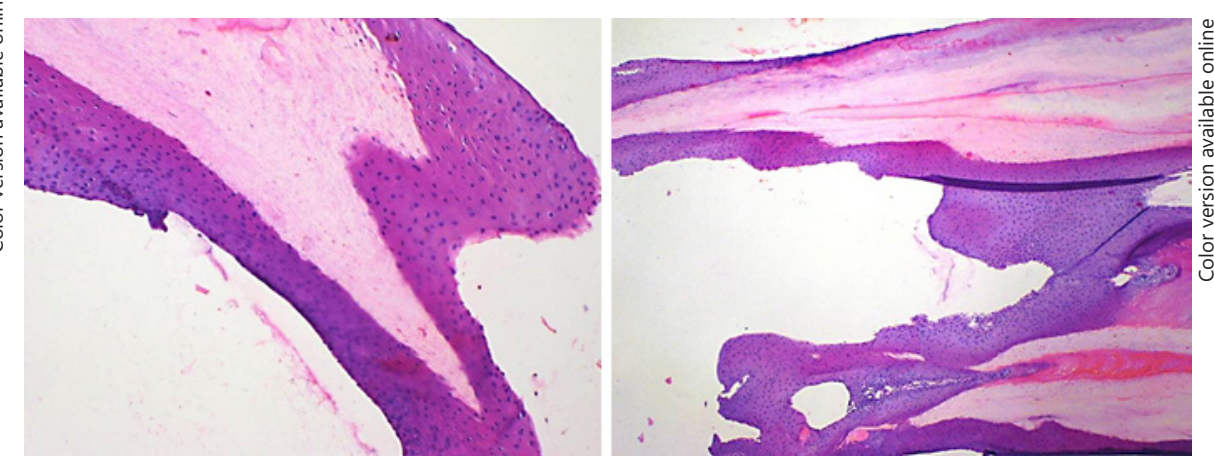

Fig. 5. Histopathological examination - findings of onychomatricoma. HE staining.

\section{Case Report}

A 36-year-old Caucasian male patient presented with yellowcolored nail thickening 4 years ago, which was initially between the medium and the proximal nail bed of the left hallux, without affection of the proximal nail fold. It was followed by progression of the lesion, affecting almost the entire nail plate in its medial region. $\mathrm{He}$ sought medical assistance at the primary health care level, where a systemic and topical treatment for onychomycosis was prescribed; however, the condition did not improve. He reported that occasionally he had small traumas in the region of the toes, related to his professional activity (toolmaker), but denied pain and inflammatory signs throughout this progression period.

Upon dermatologic examination, there was striped brown-yellowish nail thickening, dystrophy, and longitudinal hypercurvature in the lateromedial region of the left hallux nail plate (Fig. 1). Upon palpation, hardened consistency was found. The direct mycological examination was negative, and the radiographic analysis did not reveal bone changes.

We opted for performing an exploratory surgical procedure. After dissection of the proximal nail fold, we observed predominant affection of the medium and distal nail plate (Fig. 2). A careful avulsion of the nail plate evidenced parallel cavitations and orifices in its ventral face (Fig. 3a), associated with digitiform projections of the matrix as well as the proximal and medium nail bed (Fig. 3b). These cavitations and orifices fit perfectly in the tumor surface. Excision (Fig. 4) and histopathological examination (Fig. 5) of the affected nail matrix and bed were performed, which confirmed the diagnosis of onychomatricoma.

\section{Discussion}

In addition to the conventional clinical and intraoperative findings of onychomatricoma, rare forms of presentation have been described in the literature. Perrin and Baran [6], in 2008, described 3 cases that clinically presented with dorsal pterygium. These authors conducted a thorough histological analysis of the tumor area and nail plate and showed that onychomatricoma can originate in the ventral portion of the proximal nail fold. They propose the occurrence of metaplasia at the site as the pathogeny. They also suggest that some signs, such as nail plate depression, can be secondary to the tumor's compressive effect on the normal nail matrix [6].

Metaplasia is considered as a type of transdifferentiation that generates change of the cellular phenotype. It may be reversible and usually results from a lesion or chronic inflammation of a given tissue. Overall, the main examples of this transdifferentiation include Barrett's esophagus (gastroesophageal metaplasia), pulmonary squamous metaplasia, and cutaneous mucinous metaplasia [8].

To justify this form of presentation, we should also cite two recent studies that showed the possibility for the proximal matrix to have dorsal expansions towards the ventral surface of the proximal nail fold $[9,10]$. This would lead to the probability of onychomatricoma formation from these expansions, without the need for a metaplasia.

Our case showed predominant affection of the nail bed, evidenced by the intraoperative findings. Wang et al. [11] described the only case of onychomatricoma affecting the nail bed. They report the case of an adult male patient with black-colored striped nail thickening, with higher thickness in the distal region of the first left finger. There was slow, progressive growth over a period of 7 years. Histology confirmed the prominent V-shaped papillomatous proliferations of epithelial basophilic cells in the nail bed and thickening of the nail plate [11].

Histological analyses of the 2 cases corroborated the typical findings of onychomatricoma, but in the nail bed. To 
explain the origin of this nail bed tumor, the hypothesis of metaplasia with cell formation of the matrix was proposed [11]; that is, a similar pathogeny to the form with affection of the proximal nail fold, but in different topographies.

With the foregoing information, it is currently said that onychomatricoma might have its origin in the nail matrix or in areas of cell differentiation associated with the formation of matrix cells (metaplasia), which may be the ventral portion of the proximal nail fold [6] or the nail bed [11].

\section{Statement of Ethics}

The authors have no ethical conflicts to disclose. The patient provided his consent to publish photos and details of the case.

\section{Disclosure Statement}

The authors have no conflicts of interest to declare.

\section{References}

1 Baran R, Kint A. Onychomatrixoma. Filamentous tufted tumour in the matrix of a funnel-shaped nail: a new entity (report of three cases). Br J Dermatol. 1992 May;126(5):510 5

2 Tavares GT, Di Chiacchio N, Di Chiacchio NG, Souza MV. Onicomatricoma: um tumor desconhecido pelo dermatologista. An Bras Dermatol. 2015;90(2):268-70. Portuguese.

3 Perrin C, Baran R, Balaguer T, Chignon-Sicard B, Cannata GE, Petrella T, et al. Onychomatricoma: new clinical and histological features. A review of 19 tumors. Am J Dermatopathol. 2010 Feb;32(1):1-8.
4 Fayol J, Baran R, Perrin C, Labrousse F. Onychomatricoma with misleading features. Acta Derm Venereol. 2000 Sep-Oct;80(5):370-2.

5 Di Chiacchio N, Tavares GT, Tosti A, Di Chiacchio NG, Di Santis E, Alvarenga L, et al. Onychomatricoma: epidemiological and clinical findings in a large series of 30 cases. $\mathrm{Br} \mathrm{J}$ Dermatol. 2015 Nov;173(5):1305-7.

6 Perrin C, Baran R. Onychomatricoma with dorsal pterygium: pathogenic mechanisms in 3 cases. J Am Acad Dermatol. 2008 Dec;59(6): 990-4.

7 Stewart CL, Sobanko JF, Rubin AI. Myxoid onychomatricoma: an unusual variant of a rare nail unit tumor. Am J Dermatopathol. 2015 Jun;37(6):473-6.
8 Regalo G, Leutz A. Hacking cell differentiation: transcriptional rerouting in reprogramming, lineage infidelity and metaplasia. EMBO Mol Med. 2013 Aug;5(8):1154-64.

9 Perrin C, Langbein L, Schweizer J. Expression of hair keratins in the adult nail unit: an immunohistochemical analysis of the onychogenesis in the proximal nail fold, matrix and nail bed. Br J Dermatol. 2004 Aug;151(2):362-71.

10 Perrin C. Expression of follicular sheath keratins in the normal nail with special reference to the morphological analysis of the distal nail unit. Am J Dermatopathol. 2007 Dec;29(6): 543-50.

11 Wang L, Gao T, Wang G. Nail bed onychomatricoma. J Cutan Pathol. 2014 Oct;41(10):783-6. 